\title{
Fixation Differences in Visual Search of Accident Scenes by Novices and Expert Emergency Responders
}

Erik Prytz, Caroline Norén and Carl-Oscar Jonson

The self-archived postprint version of this journal article is available at Linköping University Institutional Repository (DiVA):

http://urn.kb.se/resolve?urn=urn:nbn:se:liu:diva-150343

N.B.: When citing this work, cite the original publication.

Prytz, E., Norén, C., Jonson, C., (2018), Fixation Differences in Visual Search of Accident Scenes by Novices and Expert Emergency Responders, Human Factors.

Original publication available at:

Copyright: SAGE Publications (UK and US)

http://www.uk.sagepub.com/home.nav 
Topic: Sensory and Perceptual Processes

\title{
FIXATION DIFFERENCES IN VISUAL SEARCH OF ACCIDENT SCENES BY NOVICES AND EXPERT EMERGENCY RESPONDERS
}

\author{
Erik G. Prytz ${ }^{1}$, Caroline Norén ${ }^{1}$, Carl-Oscar Jonson ${ }^{2}$ \\ ${ }^{1}$ Department of Computer and Information Science, Linköping University \\ ${ }^{2}$ Center for Disaster Medicine and Traumatology, and Department of Clinical and \\ Experimental Medicine, Linköping University
}

\begin{abstract}
Objective: This study sought to investigate whether expert-novice differences in visual search behavior found in other domains also apply to accident scenes and the emergency response domain.

Background: Emergency service professionals typically arrive at accidents only after being dispatched when a civilian witness has called an emergency dispatch number.

Differences in visual search behavior between the civilian witness (usually a novice in terms of emergency response) and the professional first responders (experts at emergency response) could thus result in the experts being given insufficient or erroneous information, which would lead them to arrive unprepared for the actual situation.

Method: A between-subjects, controlled eye-tracking experiment with 20 novices and 17 experts (rescue and ambulance service personnel) was conducted to explore expertnovice differences in visual search of accident and control images.

Results: The results showed that the experts spent more time looking at task-relevant areas of the accident images than novices, as predicted by the information reduction hypothesis. The longer time was due to longer fixation durations rather than a higher fixation count.
\end{abstract}


Conclusion: Expert-novice differences in visual search are present in the emergency domain. Given that this domain is essential to saving lives and also relies heavily on novices as the first link in the chain of response such differences deserve further exploration.

Application: Visual search behavior from experts can be used for training purposes. Eye-tracking studies of novices can be used to inform the design of emergency dispatch interviews.

\section{Keywords:}

visual search, disaster response, emergency medicine, eye tracking, expert-novice differences

\section{INTRODUCTION}

The "chain of survival" is a metaphor for the necessary steps taken by an emergency response system to ensure patient survival following an accident (Søreide, 2012). The first link in this chain is the recognition of an accident and activation of the emergency response system. Commonly, this is initiated through a call to an emergency dispatch center from an eyewitness or bystander who has observed an accident. The appropriate response to that accident is then determined by an emergency dispatch operator, who allocates the necessary resources from the emergency services, which traditionally includes the rescue services, emergency medical services, and police. The initial professional emergency service response to an accident thus relies on the information provided by the caller and the emergency dispatch operator's ability to interpret this information.

The dispatcher's task of gathering and interpreting information can be challenging (Ek \& Svedlund, 2014). The practical limitations of the dispatcher's information-gathering process contribute to a lack of information that, together with communication difficulties and insufficient resources, can create feelings of powerlessness and uncertainty for the dispatcher 
(Forslund et al., 2004). These aspects contribute to the stressful environment reported by emergency dispatchers (Weibel, Gabrion, Aussedat, \& Kreutz, 2003). The dispatcher must, in a limited time, interview the caller and determine what has happened and if it truly is an emergency. If it is an emergency, the dispatcher determines what priority the incident should be given and if there is need for a fellow operator to join the call and share the work. Finally, the dispatcher will dispatch the appropriate first responder units (Forslund, Kihlgren, \& Kihlgren, 2004; Normark, 2002). In virtually all current systems the operators are limited to verbal communication as the only source of information. From this they must construct a mental representation of the incident that the caller is reporting and dispatch the appropriate responder units (Blandford \& Wong, 2004).

The responder units are dispatched from the rescue, police, and ambulance services alone or in an appropriate combination. In incidents with multiple casualties, the number of responding units and dispatch center's assignment priority is crucial; too few responders might result in patients in critical conditions suffering adverse outcomes, but too many responders deplete the readiness to respond to other calls which also can result in adverse outcomes (Khorram-Manesh, Montán, Hedelin, Kihlgren, \& Örtenwall, 2011). The response of the professional organizations, from dispatch operators to emergency services, thus relies on the information provided by the first link: the caller.

This caller can be anyone who happens to observe an accident and has access to a telephone. Emergency numbers such as the American 911 or the European 112 are known by virtually all citizens, and citizens are generally encouraged to report any accidents they witness. Therefore, the typical caller is unlikely to be a trained professional given that only a small percentage of the population works in an emergency response profession. The information provided to the dispatchers may therefore differ from what the emergency services need to know; the caller might focus on obvious or salient cues (e.g., a car on fire) 
while not mentioning less obvious pieces of information that could be critical in the allocation of resources (e.g., a tanker with a hazardous substance symbol parked next to the car on fire). To mitigate this risk, most dispatch centers employ standardized interview protocols to guide the caller's information gathering through targeted questions. To what extent such questions influence the caller's visual search behavior, and subsequent reporting, has not been well investigated. Additionally, the extent to which the visual search behaviors of untrained callers differ from those of experts when viewing accident or emergency scenes has not been established. This knowledge gap makes it difficult to design and implement protocols to mitigate deficiencies and elicit pertinent high-quality information.

Differences in visual search patterns between novices and experts have been established in many other domains. In a meta-review of such eye-tracking studies, Gegenfurtner, Lehtinen, and Säljö (2011) contrasted three different theories that explain gaze behavior differences between novices and experts: the long-term working memory theory (LTWM; Chase \& Ericsson, 1982; Ericsson \& Kintsch, 1995), the information reduction hypothesis (IRH; Haider \& Frensch, 1996), and the holistic model of image perception (HMIP; Kundel, Nodine, Conant \& Weinstein, 2007). Briefly, the LTWM theory proposes that experts are able to use their long-term memory as an extension of their working memory due to efficient retrieval structures and revisualizations of larger visual chunks of information. This enables the experts to process more visual information in a shorter time, resulting in overall shorter fixation durations. The IRH, on the other hand, suggests that experts instead learn to focus on task-relevant cues and ignore task-irrelevant cues, enabling them to allocate their attentional resources only to the visual cues necessary for task performance. As a result, experts should show fewer or shorter fixations on task-irrelevant areas. Finally, the HMIP proposes that experts are able to process additional information 
from non-foveal areas, enabling a more rapid visual search. This would be evident in longer saccades and shorter time to first fixation on task-relevant areas.

Gegenfurtner and colleagues' (2011) meta-review, which included 296 effect sizes across 71 studies, provided support for some predictions made by these theories. Specifically, they found support for the prediction based on LTWM that experts have shorter fixation durations than novices overall, but also strong support for the prediction that experts have more fixations of longer durations on task-relevant areas as predicted by the IRH.

Additionally, experts exhibit longer saccades and shorter times to first fixate on task-relevant information as predicted by HMIP. These effects were also moderated by other factors, such as visualization characteristics (e.g., smaller effects for static rather than dynamic scenes and for 2-dimensional rather than 3-dimensional visualizations), task characteristics (e.g., smaller effects for less complex tasks and for limited rather than unlimited time-on-task), as well as the type of professional domain (e.g., sports, medicine, or transportation).

None of the studies included in Gegenfurtner et al.'s (2011) meta-review examined the emergency response domain. This domain is, however, of particular interest because of its practical implications given that "novices" are often found as the initiator of professional work. The purpose of the current study was thus to investigate if domain expertise influences visual search patterns in emergency call situations. Of the three theories described by Gegenfurtner et al., the IRH was determined to be the most applicable to this problem domain as it concerns visual search of task-relevant areas, which would correspond to searching relevant areas of an accident scene to create a meaningful and comprehensive verbal description to an emergency dispatcher. Previous studies have also shown that expert police officers have longer fixations on task-relevant areas in threatening situations as compared to rookie officers (Vickers \& Lewinski, 2012), and longer gaze duration on potential hazards during pursuit driving (Crundall, Chapman, \& Underwood, 2003; Crundall et al., 2005). 
Those findings are also in line with the IRH. Based on the IRH we therefore predicted that experts would have more fixations, as well as longer fixations, on task-relevant areas, which should result in overall more time spent looking at those areas, compared to the novices. These predictions were tested in a controlled experiment that included both novices and two types of experts (rescue service personnel and ambulance personnel).

\section{METHOD}

\section{Participants}

A power analysis based on the effect sizes reported in Gegenfurtner et al. (2011) indicated that 33 participants would be necessary to test the predictions. A total of 37 participants recruited from one region in Sweden completed the study. Of these, 20 were novices and 17 were experts. The novices were students from a local university and were recruited through disseminating information about the study on campus and via programspecific email lists. All experts had professional training and were currently active in a branch of emergency services, whereas none of the novices had previous training or experience in this domain. Nine of the experts were ambulance staff and the remaining eight were from the rescue services. The experts were recruited through professional contacts via a teaching institute for disaster and emergency medicine. The novice sample was younger (median age 24.5) than either the ambulance staff (median age 30) or rescue services (median age 35) samples. The ambulance staff had a mean professional experience of 9.7 years $(S D=$ $10.9)$, and the rescue services staff 15.4 years $(S D=12.8)$. The participants did not receive any incentive or compensation to participate in the study.

\section{Task}

The participants were asked to look at a series of images presented on a computer screen. Their task was to determine whether the presented image depicted an accident, i.e. a 
situation that would have triggered them to call the emergency dispatch had they observed it in real life. If so, they were to press the S-key on the keyboard and give a brief (max 60 seconds) verbal report that detailed the situation to a fictitious emergency dispatch. The image was visible for the entire duration of those 60 seconds, and the participant's eye movements were tracked using eye-tracking equipment. If the participants determined that the image did not depict an emergency they were to instead press the L-key, and simply observe the image for 60 seconds. Once the participants had given a response they could not change it. A gray screen was shown after each image to provide a rest and recovery period. The participants themselves triggered the presentation of the next image using the space bar. The image order was randomized for each participant. The participants were instructed to be both fast and accurate in their decision to call or not call the emergency dispatch.

\section{Apparatus}

Two devices, one stationary and one mobile, were used for the eye tracking measurements; both were of the model Remote Eye Tracking Device by Sensomotoric Instrument. The stationary version was used for the novice group as they could participate in the experiment at the campus location where this version was installed. The portable version was used for the two expert groups, as these two groups were on-call and therefore participated at their respective stations. The stationary model had a maximum data recording frequency of $500 \mathrm{~Hz}$ and the portable model $60 \mathrm{~Hz}$. For each setup, a 22-inch computer monitor was used to display the images at a 1280x1024 pixels resolution at an approximate distance of $60 \mathrm{~cm}$ from the observer. The participants were free to move their heads during the experiment but were encouraged to remain relatively still to minimize the risk of calibration errors. A second computer was connected to the experimental setup to allow the experiment leader to observe the output from the eye tracking equipment in real time and verify calibration results. Iview $\mathrm{X}^{\mathrm{TM}}$, SMI Experiment Center ${ }^{\mathrm{TM}}$ and $\mathrm{SMI} \mathrm{BeGaze}^{\mathrm{TM}}$ software 
were used to analyze the eye tracking data. Audio recordings of participant reports to emergency dispatch were also collected.

\section{Procedure}

The experiment followed the tenets of the Helsinki declaration and, as per Swedish law and customary procedure, did not require formal IRB approval. The participants were run individually. Each participant was informed about the experiment and its purpose prior to reading and signing the consent form. Following consent, participants completed a demographics questionnaire. They were then instructed on the task they were asked to perform during the experiment. The participants were also informed that they were at the scene in a non-professional capacity and did not have access to any special equipment. The participants were not given any specific structure for the report they were to deliver to the fictitious emergency dispatch but simply asked to report what they thought was important.

A five-point calibration of the eye-tracking equipment was conducted prior to the start of the actual task. After half the stimuli had been presented a second calibration was conducted to correct for any shifts in participant position. Twenty accident images and 19 control images were presented for each participant for a total of 39 images. When all images had been presented the participants were asked to complete a post-task questionnaire and were then debriefed. The post-task questionnaire contained control questions about the experimental manipulation in terms of realism of the images and if the time to view the images was too short, sufficient, or too long. In the debriefing they were informed that the majority of the images were from various emergency exercises and drills, and that all visibly injured people on all images were moulaged markers (i.e. actors with trauma makeup).

\section{Stimuli}

The 19 control images were photographs of ordinary scenes around the local city. The remaining 20 images were mainly photographs taken during various emergency exercises and 
drills, with some complementary photographs of real emergencies for which no suitable exercise photos were available $(n=3)$. The real emergencies were two fires and a traffic accident. Overall, four different categories of five images each were used: 1) fire with no visibly injured persons, 2) single injured person, 3) traffic accident with at least one visibly injured person, and 4) traffic accident with no visibly injured persons. All images that depicted an injured person were staged using moulaged actors. The accident images were selected to be visibly and clearly emergency situations. A pilot study with novices $(n=3)$ and experts $(n=2)$ was conducted to test the images. After the pilot the images that were not sufficiently clear (i.e. had less than $100 \%$ detection rate) were changed.

Figure 1 shows one of the images used in the study in the category traffic accident with at least one visibly injured person. The image is from a large-scale emergency exercise and features two moulaged actors lying on the ground, two apparent uninjured persons, and a train tanker car leaking colored liquid. The train car features a hazardous substance label showing that the liquid is flammable and a number detailing what the liquid is (336-1093, acrylonitrile). The red squares show some of the a priori identified areas of interest containing information particularly relevant to emergency dispatch. 


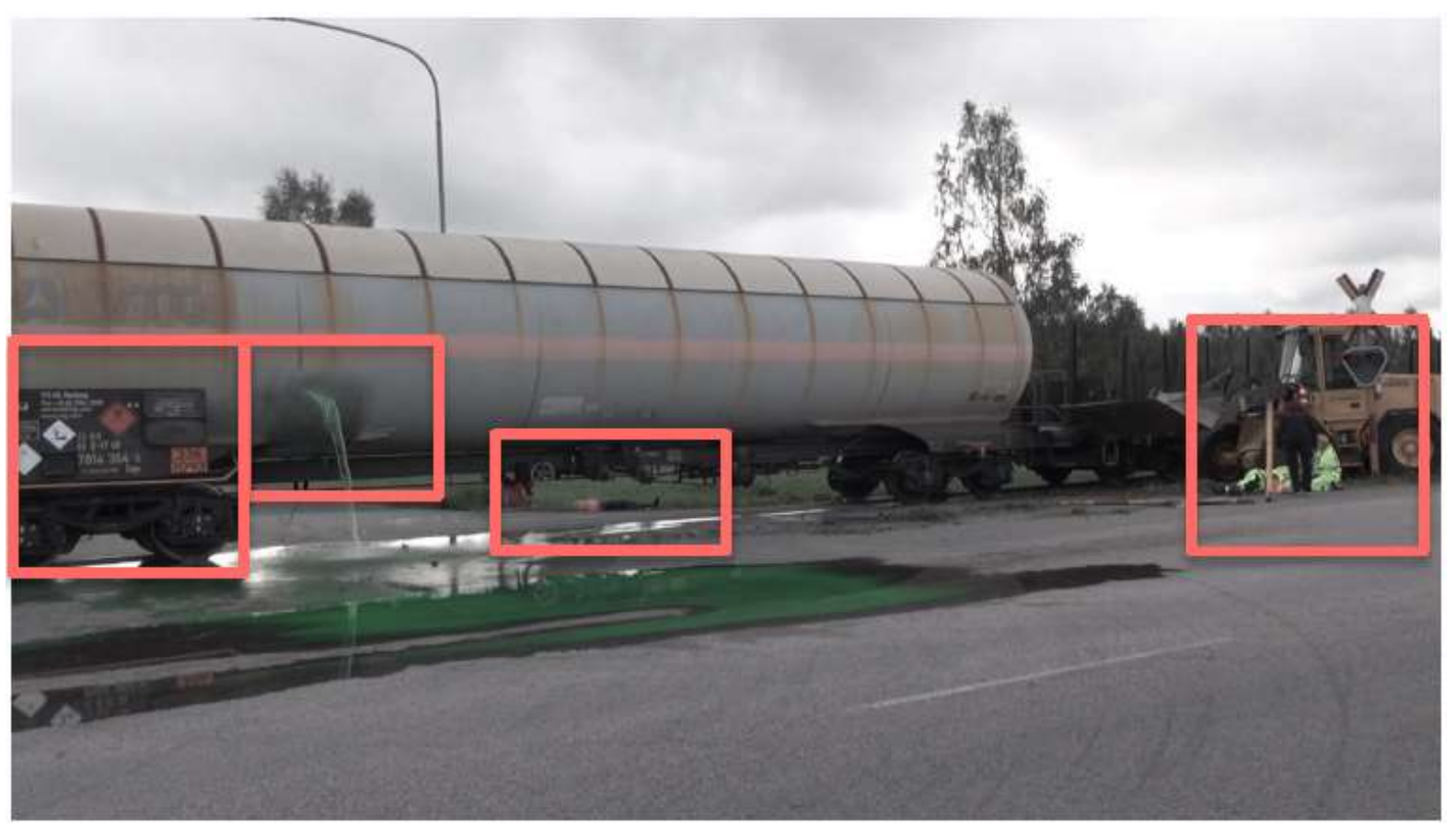

Figure 1. An image with examples of areas of interests. The areas of interest in this figure are for illustrative purposes and do not completely correspond to how the areas were defined in the eye-tracking software. The image is of lower resolution than what was presented to the participants.

\section{Areas of Interest}

Areas of interest (AOI) were determined for all images. An AOI is an informationcarrying area of the image relevant to the purpose of the task (Holmqvist et al., 2011). The AOIs for the accident images were created during a 2-hour workshop with four experts (one fire fighter and three faculty members at a teaching institution for emergency medicine who all had previous professional experience as ambulance staff). At the workshop, the participants were asked to individually mark areas of interest on each image, and then discuss in the group which areas they had marked and why. Audio data from the workshop were transcribed and subject to a content analysis to identify common categories of AOIs. These naturally differed somewhat across image categories. 


\section{Analysis}

The selected dependent eye-tracking variables were dwell time, fixation time, and number of fixations; see Table 1 for definitions. The time to decide whether the presented image depicted an accident or not was also included as decision time. The analyses for the eye-tracking variables were 3 x 4 split-plot ANOVAs with Group (novice, rescue services, ambulance) as the between-groups variable and Image Category (fire, injured person, traffic accident with injured, and traffic accident without injured) as the within-groups variable. The control image category was analyzed separately for differences across groups using 3-way ANOVAs. Planned comparisons were used to follow up main effects of group, with the first comparison being novices to experts (rescue services and ambulance combined) and the second being a comparison between the two expert groups (rescue service to ambulance). Main effects of image categories were followed up by Bonferroni-corrected pairwise comparisons. Huynh-Feldt corrections were used to correct for violations of sphericity and the corrected F-strings are reported where applicable. Decision time was analyzed using a 3 (Group) by 5 (Image category, including control) split-plot ANOVA with group as betweengroups variable and category as within-group variable. Eye tracking data from accident images where the participant chose not to call emergency dispatch were excluded from further analysis.

Table 1. Variable definitions

\begin{tabular}{llc}
\hline Variable & \multicolumn{1}{c}{ Definition } & Unit \\
\hline Decision time & Time from stimulus presentation to decision & $\mathrm{s}$ \\
Dwell time & Total time within AOIs & $\mathrm{s}$ \\
Fixation duration & $\begin{array}{l}\text { Mean fixation duration for fixations within } \\
\text { an AOI }\end{array}$ & $\mathrm{ms}$ \\
$\begin{array}{l}\text { Number of } \\
\text { fixations }\end{array}$ & Total number of fixations within AOIs & count \\
\hline
\end{tabular}




\section{RESULTS}

\section{Post-task questionnaire}

The post-task questionnaire asked the participants to rate the realism of the images from a scale of 1 (Not at all) to 7 (Very realistic). There was no significant difference in the ratings between the novices $(M=5.10, S D=1.02)$ and the experts $(M=5.47, S D=1.33)$, $t(35)=0.959, p=.344$. The questionnaire also asked on a 7-point scale if the time to view the images was too short (1), sufficient (4) or too long (7). Overall, the participants rated the time as about sufficient with no difference between the novices $(M=5.20, S D=0.95)$ and the experts $(M=5.06, S D=1.14), t(35)=0.410, p=0.684$.

\section{Decision time}

The 3 (Group) by 5 (Image category) ANOVA on decision time showed a significant interaction, Huynh-Feldt corrected $F(3.13,45.362)=4.709, p=.005$, partial $\eta^{2}=.245$, such that the novices had a shorter decision time $(M=3.10, S D=2.96)$ for the control images than the rescue service $(M=7.19, S D=3.65)$ and ambulance $(M=7.63, S D=5.73), F(2,34)=$ $5.614, p=.008$, partial $\eta^{2}=.248$. There was no difference for any of the accident categories (all $p>.315)$. In terms of accuracy, the experts had a higher hit rate $(M=98.75 \%, S D=2.88)$ than the novices $(M=94.75 \%, S D=6.17), t(34)=2.387, p=.023(95 \%$ CI $[0.59,7.40])$. The experts also had a lower miss rate $(M=0.94 \%, S D=2.01)$ as compared to the novices $(M=$ $5.25 \%, S D=6.17), t(34)=2.676, p=.011(95 \%$ CI $[-7.59,-1.04])$, but no difference in terms of false alarms $(p=.433)$ or correct rejections $(p=.835)$.

\section{Visual search behavior}

The analyses of the control image category showed no effects of group for dwell time, $F(2,34)=1.191, p=.316$, partial $\eta^{2}=.065$, mean fixation duration, $F(2,34)=2.172, p=$ .129 , partial $\eta^{2}=.113$, or number of fixations, $F(2,34)=1.520, p=.233$, partial $\eta^{2}=.082$. 
The 3 (Group) x 4 (Image Category) ANOVA for dwell time showed a main effect of image category, Huynh-Feldt corrected $F(2.292,77.925)=59.919, p<.001$, partial $\eta^{2}=$ .638 , and a main effect of group, $F(2,34)=10.449, p<.001$, partial $\eta^{2}=.381$. The image categories all significantly differed (all corrected $p \leq .024$ ) such that the traffic accident without injury category had the shortest dwell time $(M=33.64$ seconds, $S D=1.15)$, followed by the fire $(M=37.77, S D=0.81)$, traffic accident with injured $(M=42.22, S D=1.56)$, and injured $(M=53.38, S D=2.27)$ categories. The planned contrasts on group showed that the novices had significantly shorter dwell time as compared to the experts, $p<.001(95 \% \mathrm{CI}$ $[22.80,59.43])$, but no difference between the two expert groups, $p=.940$ (95\% CI [-12.97, 13.98]), see Figure 2A.

The 3 (Group) x 4 (Image Category) ANOVA for mean fixation duration showed a significant interaction, $F(6,102)=2.986, p=.010$, partial $\eta^{2}=.149$. The interaction was such that the novices had shorter mean fixation duration than the experts for all image categories: fire $(p=.010,95 \%$ CI $[20.78,141.59])$, injured $(p<.001,95 \%$ CI [80.08, 228.88]), traffic accident with injured ( $p<.001,95 \%$ CI [102.64, 222.38]), and traffic accident without injured $(p<.001,95 \%$ CI $[101.42,224.42])$. The rescue service personnel also had shorter mean fixation duration $(M=240.35, S D=73.62)$ than the ambulance personnel $(M=304.43, S D=41.08)$ for the traffic accident without injured category, $p=$ $.007,95 \%$ CI $[18.821,109.32]$, but no other category. Figure $2 \mathrm{~B}$ shows the mean fixation durations collapsed across image category. 


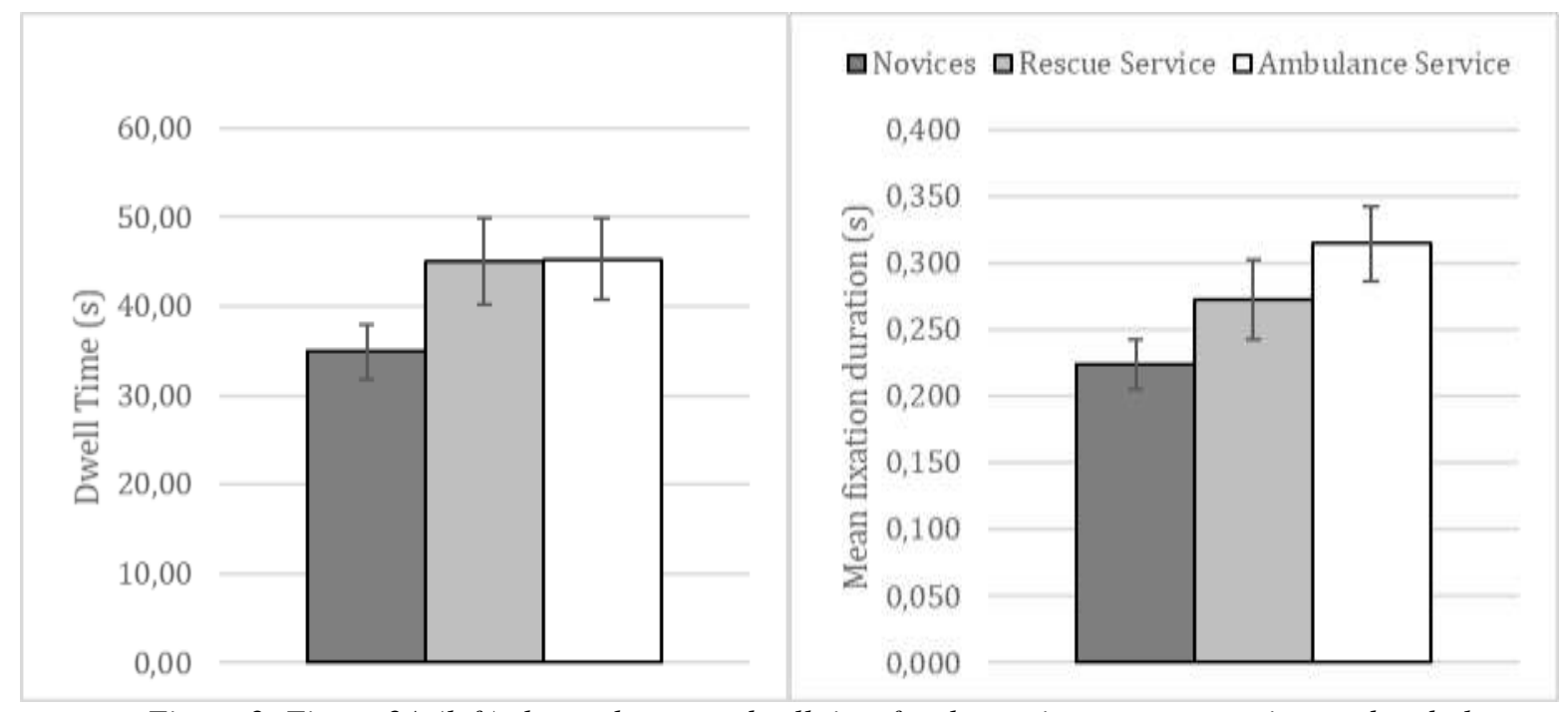

Figure 2. Figure $2 A$ (left) shows the mean dwell time for the novices, rescue service, and ambulance service collapsed across the four accident categories. Figure $2 B$ (right) shows the mean fixation duration in seconds for the novices, rescue service, and ambulance service collapsed across the four accident categories. The error bars are $95 \%$ CI.

The 3 (Group) by 4 (Image Category) ANOVA for fixation count showed a main effect of image category, Huynh-Feldt corrected $F(5.832,99.136)=18.636, p<.001$, partial $\eta^{2}=.354$, but no effect of group, $p=.116$. The image categories all significantly differed (all corrected $p \leq .022)$ except for the fire and traffic accident with injured categories $(p>.999)$, such that the traffic accident without injury category had the lowest fixation count $(M=$ $122.08, S D=3.79)$, followed by the fire $(M=137.25, S D=3.27)$ and traffic accident with injured categories $(M=138.68, S D=5.45)$, and finally the injured category $(M=154.38, S D$ $=5.36)$.

\section{DISCUSSION}

The results of the current study supported the prediction based on IRH that experts would spend more time looking at task-relevant information, as shown by longer dwell times and mean fixation durations in AOIs for experts looking at the accident images. However, the results did not support the prediction that experts should have a higher fixation count than the novices in the AOIs. The only difference between the two expert groups was that the rescue 
service group had shorter mean fixation durations as compared to the ambulance group for the traffic accident without injured category.

Novices were faster to respond to the control images than the experts. This might indicate a feature-present versus feature-absent effect (Treisman, 1989) on behalf of the experts, where they searched the entire control image for any type of "accident feature" (e.g., fire, injured person) before responding. There was no time difference for the accident images, but the experts were more accurate in their reporting with a higher hit rate and lower miss rate, although both groups scored well. This seem to indicate that an expert can make a more accurate assessment of an accident scene than a novice in the same time, which would speak to a richer mental model of accident scene features.

There are several key limitations of this study. One is that the participants conducted an unguided, exploratory search of the images. In most emergency dispatch organizations (but by no means all) the dispatch officers have access to decision support aids with questions to ask the callers. It is reasonable to believe that these questions will change the caller's visual search behavior to hunt for information that can provide an answer to the dispatch officer's question. Such an interview protocol was not included as the goal was to examine the more fundamental question of how a novice's self-guided visual search of an accident scene differs from that of an expert. It creates a baseline on which future research can built, e.g. regarding the extent to which the caller's visual search really is guided or changed by a dispatch operator's questions, or the callers' ability to extract the information required from a particular area of interest.

Another limitation is that the current study was limited to the use of static, 2dimensional images. These constraints, again based on a desire to keep the study design simple and controlled, limit the generalizability of the results to the real problem space. Follow-up studies should test the different effects of more realistic conditions such as 
dynamic (video) images in 2D or 3D, or even field studies of staged accident scenes with mobile eye-tracking equipment. Gibson's (1979) notion of ecological perception stress that perception is active and exploratory beyond merely top-down or bottom-up processing of abstract features. In line with this, Gegenfurtner et al.'s (2011) meta-review indicated that static, 2-dimensional visualizations are associated with smaller expert-novice differences, which means that it is likely that a larger visual search difference would be observed in a more dynamic, 3-dimensional setting.

A possible confound in the experiment is age, as the novice group was on average younger than the expert group. The median age in all three groups was relatively young, with the highest median being 35 for the rescue service workers, whereas most research on agerelated differences deal with significantly older populations (see for instance Madden et al., 2004; Rogers, Fisk, \& Hertzog, 1994; Scialfa, \& Joffe, 1997). The confound of age in the current study cannot be completely discounted, but the lack of differences for the control images suggest that any potential age-related effect was likely small. Related, the two expert groups had a rather large standard deviation for years of professional experience. This likely reflects the fact that both young as well as older professionals were included in the study. Future studies might wish to control for or exclude professionals with little professional experience.

This study did not test the predictions based on the HMIP theory that experts should have longer saccades and shorter time to first focus on task-relevant areas. These variables were not included for two reasons. First, they were deemed less relevant to the particular task and problem setting in this study. The longer decision time by the experts for the control images supports this notion with regards to time to first fixation, indicating that the first fixation time is less relevant than a comprehensive search to accomplish the task. Second, the difference in equipment setup, particularly the recording frequency, could introduce 
measurement errors when analyzing saccades. Thus, this study was limited to focus on the overall AOI-relevant variables that were deemed more informative for the real domain problem.

In summary, this study explored the information-gathering problems related to the fact that novices often are the initiators of a complex emergency response chain by calling an emergency dispatch such as 911 . The results of this study support the hypothesis that the typical emergency dispatch caller will differ in their visual information gathering process compared to the professionals who will later arrive at the scene. In an emergency response context, the results imply that the likely emergency dispatch callers will spend less time looking at features containing information relevant to the responders' mission. A practical implication of these results is therefore as support for the use of standardized emergency dispatch interview guides that can direct the caller's attention to useful information. Although this is standard practice in most industrialized countries it is by no means so globally. The results can also be used to inform the design of new interview guides and serve as a baseline to test against. The extent to which current interview guides already used in many modern emergency dispatch systems direct the attention of the caller is not known and should be given more attention in future studies. The results from this study also indicate new opportunities and issues in need of scientific attention when emergency dispatch centers start accepting emergency calls that includes video. In this future, plausible scenario, emergency dispatch operators must be trained to guide the caller's video recording, and to select relevant episodes to push to the responders en route. Knowing what professional first responders look for will be beneficial also for that purpose.

\section{Précis (50 words in 1-3 sentences describing the manuscript):}

Presently, no study has examined expert-novice differences in visual search in the emergency response domain. This domain is relevant given the critical role novices play in 
observing and reporting accidents to dispatchers. This study demonstrates expert-novice differences for visual search of accident scenes and provides a baseline for further research.

\section{KEY POINTS}

- Expert-novice differences in visual search have been found in several domains, but have not been explored in the emergency response domain

- This study showed that experts have longer dwell times and fixation durations on task-relevant areas of accident images, as compared to novices

- The results from this study can be helpful to in the design of new emergency dispatch interview protocols

\section{ACKNOWLEDGMENT}

We would like to thank the participants who volunteered their time to this study, as well as the helpful comments on the manuscript provided by our colleagues and the three anonymous reviewers. In particular, Dr. Rachel Phillips, Dr. Johan Berlin, and Dr. Eric Carlström provided helpful input at different points of the project. This research was funded by the Swedish Civil Contingencies Agency (MSB) [Grant 2011-4957].

\section{REFERENCES}

Blandford, A., \& Wong, B. L. W. (2004). Situation awareness in emergency medical dispatch. International Journal of Human Computer Studies 61, 421-452. https://doi.org/10.1016/j.ijhcs.2003.12.012

Chase, W. G., \& Ericsson, K. A. (1982). Skill and working memory. In G. H. Bower (ed.), The psychology of learning and motivation. New York, NY: Academic Press.

Crundall, D., Chapman, P., France, E., Underwood, G., \& Phelps, N. (2005). What attracts attention during police pursuit driving? Applied Cognitive Psychology, 19(4), 409-420. https://doi.org/10.1002/acp.1067 
Crundall, D., Chapman, P., Phelps, N., \& Underwood, G. (2003). Eye Movements and Hazard Perception in Police Pursuit and Emergency Response Driving. Journal of Experimental Psychology: Applied, 9(3), 163-174. https://doi.org/10.1037/1076898X.9.3.163

Ek, B., \& Svedlund, M. (2014). Registered nurses' experiences of their decision-making at an Emergency Medical Dispatch Centre. Journal of Clinical Nursing, 24, 1122-1131.

Ericsson, K. A., \& Kintsch, W. (1995). Long-Term Working Memory. Psychological Review, 102(2), 211-245.

Forslund, K., Kihlgren, A., \& Kihlgren, M. (2004). Operators' experiences of emergency calls. Journal of Telemedicine and Telecare, 10(5), 290-297. https://doi.org/10.1258/1357633042026323

Gegenfurtner, A., Lethinen, E., \& Sälsjö, R. (2011). Expertise differences in the comprehension of visualizations: A meta-analysis of eye-tracking research in professional domains. Educational Psychology Review, 23, 523-552.

Gibson, J. J. (1979). The ecological approach to visual perception. Boston, MA: Houghton Miffin.

Haider, H., \& Frensch, P. A. (1996). The role of information reduction in skill acquisition. Cognitive Psychology, 30, 304-337.

Holmqvist, K., Nyström, M., Andersson, R., Dewhurst, R., Jarodzka, H., \& van de Weijer, J. (2011). Eye tracking: A Comprehensive Guide to Methods and Measures. New York, NY: Oxford University Press Inc.

Khorram-Manesh, A., Montán, K. L., Hedelin, A., Kihlgren, M., \& Örtenwall, P. (2011). Prehospital triage, discrepancy in priority-setting between emergency medical dispatch centre and ambulance crews. European Journal of Trauma and Emergency Surgery, 37(1), 73-78. https://doi.org/10.1007/s00068-010-0022-0

Kundel, H. L., Nodine, C. F., Conant, E. F., \& Weinstein, S. P. (2007). Holistic component of image perception in mammogram interpretation: Gaze-tracking study. Radiology, 242(2), 396-402.

Madden, D. J., Whiting, W. L., Cabeza, R., \& Huettel, S. A. (2004). Age-related preservation of top-down attentional guidance during visual search. Psychology and aging, 19(2), 304.

Normark, M. (2002). Sense-making of an emergency call. In Proceedings of the second Nordic conference on Human-computer interaction - NordiCHI '02 (p. 81). New York, New York, USA: ACM Press. https://doi.org/10.1145/572020.572031

Rogers, W. A., Fisk, A. D., \& Hertzog, C. (1994). Do ability-performance relationships differentiate age and practice effects in visual search?. Journal of Experimental Psychology: Learning, Memory, and Cognition, 20(3), 710. 
Scialfa, C. T., \& Joffe, K. M. (1997). Age differences in feature and conjunction search: Implications for theories of visual search and generalized slowing. Aging, Neuropsychology, and Cognition, 4(3), 227-246.

Søreide, K. (2012). Strengthening the trauma chain of survival. British Journal of Surgery, 99(S1), 1-3. https://doi.org/10.1002/bjs.7795

Treisman, A. (1986). Features and objects in visual processing. Scientific American, 255(5), 114-125. doi: 10.1038/scientificamerican1186-114B

Vickers, J. N., \& Lewinski, W. (2012). Performing under pressure: Gaze control, decision making and shooting performance of elite and rookie police officers. Human Movement Science, 31(1), 101-117. https://doi.org/10.1016/j.humov.2011.04.004

Weibel, L., Gabrion, I., Aussedat, M., \& Kreutz, G. (2003). Work-related stress in an emergency medical dispatch center. Annals of Emergency Medicine, 41(4), 500-506. https://doi.org/10.1067/mem.2003.109

\section{Author biographies:}

Erik G. Prytz at the Department of Computer and Information Science, Linköping University, received his $\mathrm{PhD}$ in Human Factors Psychology from Old Dominion University in 2014 .

Caroline Norén obtained her MSc in Cognitive Science from Linköping University in 2016.

Carl-Oscar Jonson at the Center for Teaching and Research in Disaster Medicine and Traumatology, Linköping University received his $\mathrm{PhD}$ in Medicine from Linköping University in 2007. 